\title{
COMMISSION 29: STELLAR SPECTRA
}

\section{(SPECTRES STELLAIRES)}

\author{
PRESIDENT: G. Mathys \\ VICE-PRESIDENT: C. Sneden \\ ORGANIZING COMMITTEE: B. Barbuy, E. Friel, R. Gratton, U. Heber, \\ J.D. Landstreet, P. Molaro, P.E. Nissen, M. Parthasarathy, N. Piskunov, \\ F. Primas \& F. Spite
}

\section{Introduction}

Access to new generation $8-10 \mathrm{~m}$ class telescopes and to their efficient spectrographs becomes increasingly widespread and opens a wealth of new possibilities for stellar spectroscopy. The advent of high-resolution spectrographs with very good throughput down to the UV atmospheric cutoff, such as UVES at ESO's VLT, allows unprecedented precision to be achieved in the analysis of important diagnostic lines that could hardly be exploited so far, leading to major advances in studies of very metal-poor stars, light elements, and r- and s-process products. Spectroscopic studies of subtle effects such as magnetic fields and stellar pulsation are boosted by the combination of high signal-to-noise and high time resolution that has become achievable for the first time. This is also the start of the era of high-resolution studies of individual stars in external galaxies.

\section{Late-Type Stars}

For the first time in a star with $[\mathrm{Fe} / \mathrm{H}]<-3.0$, uranium was detected, allowing the use of the U/Th ratio as a cosmic chronometer (Cayrel et al. 2001b; Hill et al. 2002).

Discrepancies between determinations of the oxygen abundance in old stars from various diagnostic lines $\left(\mathrm{OH}\right.$, forbidden $\left.[\mathrm{O} \mathrm{I}], \mathrm{O}_{\mathrm{I}}\right)$ may be solved by use of 3-D LTE hydrodynamical model atmospheres (Asplund \& García Pérez 2001) rather than 1-D models, but departures from LTE, if present, might question this conclusion. Similar discrepancies also seem to be found for sulphur (Israelian \& Rebolo 2001; Takada-Hidai et al. 2002; Nissen et al. 2002a). The behaviour of $[\mathrm{O} / \mathrm{Fe}]$ at low metallicities remains strongly debated, with apparently irreconcilable results obtained by different groups (e.g., on the one hand, Boesgaard et al. 1999, Israelian et al. 2001; on the other, Balachandran et al. 2001, Cayrel et al. 2001a, Meléndez \& Barbuy 2002, Nissen et al. 2002b; see also Depagne et al. 2002).

Heavy element abundance determinations in very metal-poor stars imply the existence of various r-process sites as well as the contribution of s-process (Burris et al. 2000; Hill et al. 2000; Sneden et al. 2000). The discovery of large amounts of lead in three metal-poor stars (van Eck et al. 2001) is consistent with the theory of s-process production. However, an alternative scenario may be operating in the extremely metal-poor star LP 625-44 (Aoki et al. 2000).

Accurate abundance determinations in the ultra-metal-poor star CS 22949-37 suggest that it exhibits the ejecta of a single supernova (Depagne et al. 2002).

New constraints on the impact of diffusion in stellar models are derived from precise abundance determinations in cluster stars, such as the studies of $[\mathrm{Fe} / \mathrm{H}]$ (Gratton et al. 2001 ) and $[\mathrm{Li} / \mathrm{H}]$ (Bonifacio et al. 2002) in NGC 6397. The widely accepted view that deep mixing in globular cluster giants is responsible for $\mathrm{O}-\mathrm{Na}$ anticorrelation is ruled out by the observation that this anticorrelation is also present in main sequence stars of NGC 6752 
that are too cold to complete the CNO cycle (Gratton et al. 2001). An alternative scenario of external origin of CNO-processed material is supported by observations of $\mathrm{C}$ and $\mathrm{N}$ in M 5 (Cohen et al. 2002). Measurements of ${ }^{12} \mathrm{C} /{ }^{13} \mathrm{C}$ in field metal-poor stars (Gratton et al. 2000) support the existence of a second mixing episodE in those stars after the RGB bump.

The existence of a population of rather fast rotators among blue horizontal branch stars cooler than $\sim 11500 \mathrm{~K}$ is confirmed (Behr et al. 2000a, b; Recio-Blanco et al. 2002). Strong chemical anomalies are present in hotter stars.

\section{Intermediate-Type Stars}

Based on systematic determinations of the projected equatorial velocities of all bright A and B stars, Abt (2000) and Abt et al. (2002) conclude from statistical arguments that rotation alone can explain the appearance of an A or late B star as either abnormal or normal.

Systematic abundance analyses in a representative sample of $\lambda$ Boo stars define a characteristic abundance pattern for this group of stars: $\sim 1$ dex deficiency of heavy elements, solar abundant light elements, and star-to-star scatter twice as large as among normal A stars (Heiter 2002). Study of two $\lambda$ Boo SB2 systems suggest that $\lambda$ Boo stars can be found at very different stages of main-sequence evolution (Iliev et al. 2002).

Based on the observation that the profiles of $\mathrm{H} \alpha$ and $\mathrm{H} \beta$ in cool Ap stars are anomalous, Cowley et al. (2001) argue that the atmospheres of these stars do not have a normal structure, contrary to a notion that had long been held. Various studies establish convincingly the existence of abundance stratification in the atmospheres of Ap stars (Bagnulo et al. 2001; Ryabchikova et al. 2002). Kochukhov \& Ryabchikova (2001a) detected a phase shift between radial velocity variations, due to pulsation, of singly and doubly ionised rare earths in the rapidly oscillating Ap (roAp) star $\gamma \mathrm{Equ}$, which is consistent with the existence of such stratification. They also observed for the first time line profile variations in this star, and in two other roAp stars (Kochukhov \& Ryabchikova 2001b). Slowly rotating Ap stars generally have their magnetic and rotation axes nearly aligned, while the angle between the two axes is predominantly large in short period Ap stars (Landstreet \& Mathys 2000). High-quality measurements of linear polarisation of spectral lines in Ap stars have been obtained for the first time, providing constraints on the transverse component of their magnetic fields (Wade et al. 2000).

Observation of variations of the spectral line $\mathrm{Hg}$ II $\lambda 3984$ in $\alpha$ And provides the first direct indication of inhomogeneities on the surface of a HgMn star (Adelman et al. 2002). Lines of Pr and Nd have been identified in the spectra of HgMn (and Am) stars; the derived elemental abundances set new constraints on the diffusion theory (Dolk et al. 2002). Underabundances of Ne diagnosed in HgMn stars set a stringent upper limit on the wind of those stars (Dworetsky \& Budaj 2000).

Lines of $\mathrm{P}$ II, Mn II, $\mathrm{Cr}$ II, $\mathrm{Ti}$ II and $\mathrm{Hg}$ II observed in emission in high resolution, high S/N spectra of normal late-B stars, $\mathrm{HgMn}$ stars and ${ }^{3} \mathrm{He}$ stars may reflect elemental stratification in their atmospheres or arise from a selective excitation process (Wahlgren \& Hubrig 2000; Sigut 2001a, b).

\section{Early-Type Stars}

The non-detection of line profile variations in early-type Be stars of the SMC may indicate that the variability of such stars in the SMC and in the Galaxy is different (Baade et al. 2002).

Magnetic fields have been detected for the first time in an $\mathrm{O}$ star $\left(\theta^{1}\right.$ Ori $\mathrm{C}$, Donati et al. 2002) and in a Be star ( $\beta$ Cep, Donati et al. 2001), which are consistent with and provide a natural interpretation of various observed properties of these stars. 
Analysis of multi-wavelength observations of $O$ stars (including far-UV FUSE data) with line-blanketed, hydrodynamic, extended non-LTE model atmospheres yield effective temperatures 15-20\% lower than most values previously derived for those stars (Crowther et al. 2002b; Bianchi \& Garcia 2002). A similar approach leads to an upward revision of the luminosity of WC4 stars in the LMC by up to 0.4 dex (Crowther et al. 2002a).

CNO abundances determined in O-type stars (Daflon et al. 2001; Villamariz et al. 2002) are consistent with the predictions of models of evolution with rotation. Determinations of abundances of oxygen in early B supergiants in M33, and of correlated Si abundances, confirm the existence of a large radial gradient of $\mathrm{O}$ abundance, much steeper than in the Milky Way (Monteverde et al. 2000).

\section{Evolved Stars}

Study of the s-process abundance distribution in $21 \mu \mathrm{m}$ carbon rich post-AGB stars (van Winckel \& Reyniers 2000) shows that the 3rd dredge-up efficiency is closely related to the strength of the integrated neutron irradiation.

Radial velocity and line profile variations with frequencies similar to those of photometric variations have been observed in several subdwarf B stars (O'Toole et al. 2000; Jeffery \& Pollacco 2000; Woolf et al. 2002), representing the first direct observations of surface motion arising from multimode non-radial oscillations in such stars.

Observation of the infrared spectrum of the extremely cool white dwarf WD0346+246 confirms model predictions according to which cool white dwarfs are much bluer than previously supposed (Hodgkin et al. 2000). The most massive double degenerate star known so far has been discovered as part to the ESO SN Ia Progenitor SurveY (SPY project); it has a mass only $10 \%$ below the Chandrasekhar limit (Napiwotzki et al. 2002). The first magnetic DZ white dwarf, LHS 2534, has been discovered by Reid et al. (2001).

Acknowledgments. Invaluable input for preparation of this report has been provided by various members of the Organising Committee of Commission 29: R. Gratton, J. D. Landstreet, P. Molaro, P. E. Nissen, M. Parthasarathy, F. Primas, and F. Spite. These contributions, which have been most helpful, are gratefully acknowledged.

Gautier Mathys

President of the Commission

\section{References}

Abt, H. A. 2000, ApJ 544, 933

Abt, H. A., Levato, H., \& Grosso, M. 2002, ApJ 573, 359

Adelman, S. J., Gulliver, A. F., Kochukhov, O. P., \& Ryabchikova, T. A. 2002, ApJ 575, 449

Aoki, W., Norris, J. E., Ryan, S. G., Beers, T. C., \& Ando, H. 2000, ApJ 536, L97

Asplund, M., \& García Pérez, A. E. 2001, A\&A 372, 601

Baade, D., Rivinius, T., Stefl, S., \& Kaufer, A. 2002, A\&A 383, L31

Bagnulo, S., Wade, G. A., Donati, J.-F., et al. 2001, A\&A369, 889

Balachandran, S. C., Carr, J. S., \& Carney, B. W. 2001, NewAR 45, 529

Behr, B. B., Cohen, J. G., \& McCarthy, J. K. 2000a, ApJ 531, L37

Behr, B. B., Djorgovski, S. G., Cohen, J. G., et al. 2000b, ApJ 528, 849

Bianchi, L., \& García, M. 2002, ApJ 581, in press

Bonifacio, P., Pasquini, L., Spite, F., et al. 2002, A\&A 390, 91

Boesgaard, A. M., King, J. R., Deliyannis, C. P., \& Vogt, S. S. 1999, AJ 117, 492

Burris, D. L., Pilachowski, C. A., Armandroff, T. E., et al. 2000, ApJ 544, 302 
Cayrel, R., Andersen, J., Barbuy, B., et al. 2001a, NewAR 45, 533

Cayrel, R., Hill, V., Beers, T. C., et al. 2001b, Nature 409, 691

Cohen, J. G., Briley, M. M., \& Stetson, P. B. 2002, AJ 123, 2525

Cowley, C. R., Hubrig, S., Ryabchikova, T. A., et al. 2001, A\&A 367, 939

Crowther, P. A., Dessart, L., Hillier, D. J., Abbott, J. B., \& Fullerton, A. W. 2002a, A\&A 392,653

Crowther, P. A., Hillier, D. J., Evans, C. J., et al. 2002b, ApJ 579, in press

Daflon, S., Cunha, K., Butler, K., \& Smith, V. V. 2001, ApJ 563, 325

Depagne, E., Hill, V., Spite, M., et al. 2002, A\&A 390, 187

Dolk, L., Wahlgren, G. M., Lundberg, H., et al. A\&A 385, 111

Donati, J.-F., Babel, J., Harries, T. J., et al. 2002, MNRAS 333, 55

Donati, J.-F., Wade, G. A., Babel, J., et al. 2001, MNRAS 325, 1265

Dworetsky, M. M., \& Budaj, J. 2000, MNRAS 318, 1264

Gratton, R. G., Sneden, C., Carretta, E., \& Bragaglia, A. 2000, A\&A 354, 169

Gratton, R. G., Bonifacio, P., Bragaglia, A., et al. 2001, A\&A 369, 87

Heiter, U. 2002, A\&A 381, 959

Hill, V., Barbuy, B., Spite, M., et al. 2000, A\&A 353, 557

Hill, V., Plez, B., Cayrel, R., et al. 2002, A\&A 387, 560

Hodgkin, S. T., Oppenheimer, B. R., Hambly, N. C., et al. 2000, Nature 403, 57

Iliev, I. Kh., Paunzen, E., Barzova, I. S. et al. 2002, A\&A 381, 914

Israelian, G., \& Rebolo, R. 2001, ApJ 557, L43

Israelian, G., Rebolo, R., García López, R. J., et al. 2001, ApJ 551, 833

Jeffery, C. S., \& Pollacco, D. 2000, MNRAS 318, 974

Kochukhov, O., \& Ryabchikova, T. 2001a, A\&A 374, 615

Kochukhov, O., \& Ryabchikova, T. 2001b, A\&A 377, L22

Landstreet, J. D., \& Mathys, G. 2000, A\&A 359, 213

Meléndez, J., \& Barbuy, B. 2002, ApJ 575, 474

Monteverde, M. I., Herrero, A., \& Lennon, D. J. 2000, ApJ 545, 813

Napiwotzki, R., Koester, D., Nelemans, G., et al. 2002, A\&A 386, 957

Nissen, P. E., Chen, Y. Q., Asplund, M., \& Pettini, M. 2002a, in IAU Symp. 210, astro$\mathrm{ph} / 0207163$

Nissen, P. E., Primas, F., Asplund, M., \& Lambert, D. L. 2002b, A\&A 390, 235

O'Toole, S. J., Bedding, T. R., Kjeldsen, H., et al. 2000, ApJ 537, L53

Recio-Blanco, A., Piotto, G., Aparicio, A., \& Renzini, A. 2002, ApJ 572, L71

Reid, I. N., Liebert, J., \& Schmidt, G. D. 2001, ApJ 550, L61

Ryabchikova, T., Piskunov, N., Kochukhov, O., et al. 2002, A\&A 384, 545

Sigut, T. A. A. 2001a, ApJ 546, L115

Sigut, T. A. A. 2001b, A\&A 377, L27

Sneden, C., Cowan, J. J., Ivans, I. I., et al. 2000, ApJ533, L139

Takada-Hidai, M., Takeda, Y., Sato, S., et al. 2001, ApJ 573, 614

van Eck, S., Goriely, S., Jorissen, A., \& Plez, B. 2001, Nature 412, 793

van Winckel, H., \& Reyniers, M. 2000, A\&A 354, 135

Villamariz, M. R., Herrero, A., Becker, S. R., \& Butler, K. 2002, A\&A 388, 940

Wade, G. A., Donati, J.-F., Landstreet, J. D., \& Shorlin, S. L. S. 2000, MNRAS 313, 823

Wahlgren, G. M., \& Hubrig, S. 2000, A\&A 362, L13

Woolf, V. M., Jeffery, C. S., \& Pollacco, D. L. 2002, MNRAS 329, 497 